\title{
A Follow-up Study of a Successful Assistive Technology for Children with ADHD and Their Families
}

\author{
Tobias Sonne $^{1}$, Paul Marshall ${ }^{2}$, Jörg Müller ${ }^{1}$, Carsten Obel $^{3}$, Kaj Grønbæk ${ }^{1}$ \\ ${ }^{1}$ Department of \\ Computer Science, \\ ${ }^{2}$ UCL Interaction Center \\ ${ }^{3}$ Department of \\ University College London, \\ Public Health, \\ Aarhus University, Denmark London, WC1E 6BT, UK Aarhus University, Denmark \\ \{tsonne, joerg.mueller, kgronbak\}@cs.au.dk, paul.marshall@ucl.ac.uk,co@au.dk
}

\begin{abstract}
No research on assistive technologies for families of children with attention deficit hyperactivity disorder (ADHD) has investigated the long-term impact after the assistive technology is returned to the researchers. In this paper, we report the outcomes of a follow-up study, conducted four-weeks after a field study of 13 children with ADHD and their families who used an assistive technology designed to help establish and change family practices. We show that some of the positive effects on parent frustration level and conflict level around morning and bedtime routines that we observed in the first phase of the study, continued even after the technology was taken away from the families. We furthermore present insights into family practices in families of children with ADHD and how these can lead to unexpected challenges and implications related to the adoption, use, and outcome of the assistive technology.
\end{abstract}

\section{Author Keywords}

Children, ADHD, attention deficit hyperactivity disorder, study design, evaluation, follow-up study, assistive technology, family practices, sleep.

\section{ACM Classification Keywords}

H.5.m. Information interfaces and presentation (e.g., HCI): Miscellaneous.

\section{INTRODUCTION}

Researchers within the IDC community have for some years explored the potential of using technology to assist children with a variety of conditions (e.g., [11,25]). However, to date only limited research has focused on children with attention deficit hyperactivity disorder (ADHD), although ADHD is the most prevalent reported mental health diagnosis for children and teens [17], with a

Paste the appropriate copyright/license statement here. ACM now supports three different publication options:

- ACM copyright: ACM holds the copyright on the work. This is the historical approach.

- License: The author(s) retain copyright, but ACM receives an exclusive publication license.

- Open Access: The author(s) wish to pay for the work to be open access. The additional fee must be paid to ACM.

This text field is large enough to hold the appropriate release statement assuming it is single-spaced in Times New Roman 8-point font. Please do not change or modify the size of this text box.

Each submission will be assigned a DOI string to be included here. worldwide prevalence of approximately 5\% [18].

ADHD is a heterogeneous disorder that manifests and affects children to varying degrees. However, the core symptoms are a combination of inattention, hyperactivity and impulsivity. Some of the common reported consequences of ADHD includes social and academic impairments [15], bedtime resistance [10], and increased risk of criminal convictions in adulthood [5]. Furthermore, ADHD has been shown to significantly affect children's quality of life: for example in one study $70 \%$ of third graders with ADHD reported that they have no close friends [28]. Within the EU, parent training is often recommended before medical treatment [30], highlighting the importance and the effect of "healthy" family/parent practices in families of children with ADHD.

Another field, which is maybe even more underexplored than ADHD within the IDC community is support for sleep. While several research projects in the broader HCI community have explored the potential of using technology to educate and assist adults in establishing healthy sleep habits (e.g. [2,12]), there does not seem to be reported similar studies for children. Nevertheless, sleep is especially important for children as poor or insufficient sleep can negatively impact learning, memory and general school performance [6].

Due to the serious negative effect of poor sleep together with the high percentage of children and teens with ADHD who suffer from sleep problems $(25-50 \%$ according to [10]), we designed a mobile system to assist families of children with ADHD in establishing healthy morning and bedtime routines: MOBERO. In the first phase of our study, MOBERO was initially evaluated with 13 children with ADHD and their families. This one-month study was divided into a two-week baseline period and a two-week intervention period where the families used MOBERO as reported in [23]

In this paper, we report on new findings from the second phase of our MOBERO study, a one-month follow-up study. Furthermore, we report on insights into family practices and unexpected outcomes of these from both the first and second phase of the study. 


\section{RELATED WORK}

\section{Assistive Sleep Technology}

Research within personal informatics and related fields has recently explored the potential of using technology to assist in improving and educating adults about their sleep habits (e.g., [2, 12]). Research within this field has focused on exploring various ways of assisting adults in improving their sleep habits; ShutEye [2] uses a mobile approach where the wallpaper of the user's mobile phone provides insights into the sleep impact of certain activities, such as consuming food and beverages at different times: e.g. drinking coffee after 8pm might negatively effect sleep quality. Similarly, Lullaby [12] uses a range of environmental sensors, and a tablet display to educate and assist users in creating optimal conditions for sleep.

Even though poor sleep habits negatively affect children [6], no research within the HCI community has yet explored the potential for technology to support children's sleep.

\section{Assistive Technology for Children with ADHD}

In contrast to the research on sleep technologies, HCI researchers have recently begun to report early research on assistive technologies for children with ADHD [21], including TangiPlan [29], ChillFish [22], BlurtLine [20], and CASTT [24].

TangiPlan was one of the first examples of research that explored the potential of using technology to support children with ADHD [29]. TangiPlan is a tangible system that assists children with ADHD in completing their morning routines as a way to improve their executive functioning [29]. ChillFish [22] is a biofeedback game designed to assist children with ADHD in performing a calming breathing exercise. By breathing through an physical LEGO fish embedded with electronics the player controls the player character's movement in the ChillFish game, and though an strategic placement of star fish (points in the game) the player is obliged to use a relaxing breathing rate. BlurtLine [20] and CASTT [24] are both examples of technologies, which in contrast to TangiPlan and ChillFish focus on providing sensor-based assistance in-situ to children with ADHD in school contexts. BlurtLine is designed to assist the child to avoid blurting (impulsive speaking behavior), which is achieved by using an interactive chest strap that works both as a sensor (in detecting forthcoming blurts) and as intervention device by providing the child with tactile feedback [20]. In contrast, CASTT focuses on assisting children with ADHD to regain and maintain attention during school lessons. CASTT relies on worn accelerometers to detect when the child has lost attention, and a mobile phone application to assist them in regaining it [24].

TangiPlan, ChillFish BlurtLine and CASTT are all examples of technologies that have potential to assist and empower children with ADHD. However, research is mostly based on early prototypes and the evaluations are limited in either number of participants, duration or both.

We advance the state of the art of technologies that support sleep for adults by highlighting the potential for designing for children with ADHD. Furthermore, we build upon the limited existing work within the domain of assistive technologies for children with ADHD by investigating the prolonged effect of using an assistive technology after an initial field study, and after this technology has been taken away from the families. Finally, we contribute with insights on designing for families of children with ADHD.

\section{MOBERO - A MOBILE TOOL TO ESTABLISH HEALTHY MORNING AND BEDTIME ROUTINES}

MOBERO (presented in more detail in [23]) is a mobile application that assists parents and children with ADHD to establish healthy morning and bedtime routines, as these situations can often cause frustration $[8,19]$. We designed and developed MOBERO though an iterative design process involving parents, children with ADHD and ADHD domain professionals as described in [23]. As ADHD is highly hereditable [3] and because we learned from our empirical studies that parents' engagement was critical in changing children's morning and bedtime routines, we included routines for parents as well as children in MOBERO [23].

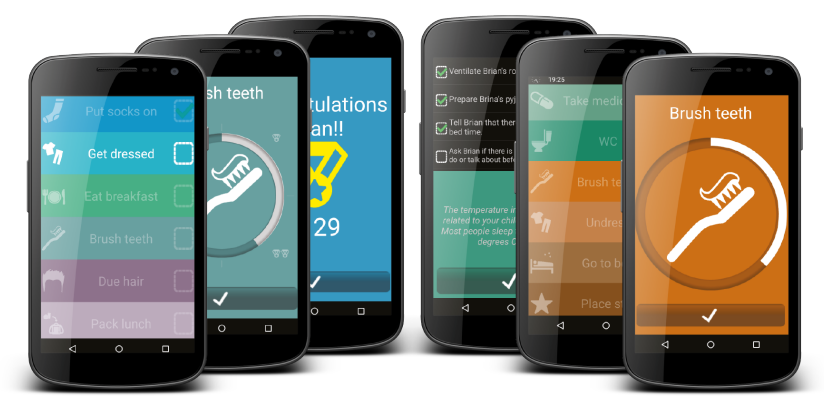

Figure 1: The MOBERO mobile system. Left, the morning module. Right, the bedtime module.

MOBERO consist of two modules; a bedtime module and a morning module. However, in the following we will refer to both as simply MOBERO. Around bedtime MOBERO notifies the parents to start their bedtime routine approximately 15-20 minutes before the child is expected to go to bed, which is discussed and agreed with the parent prior to them starting to use MOBERO. The parent routine includes activities like ventilating the child's bedroom, preparing the child's pajamas, and notifying the child about the upcoming bedtime. The intention of including these parent activities is to remind the parents about their child's upcoming bedtime and to provide optimal conditions for sleep. After the parents have completed their bedtime routine, MOBERO displays a 15 minutes' countdown timer, after which it alerts the child to begin her bedtime routines. Though a structured list of icons and text, the child is guided through each activity as illustrated in Figure 1. 


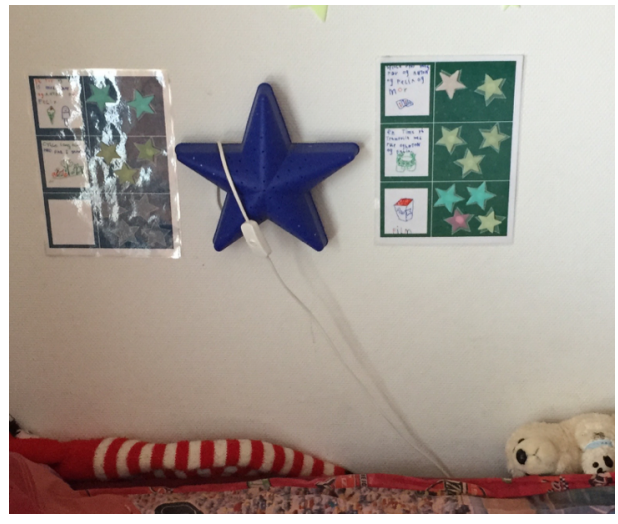

Figure 2: Two A4-sized paper reward charts placed above a child's bed. The child and parents agree on a reward, which the child then draws in the "white boxes". When the child has collected the amount of stars that each reward is worth (two, three, or four stars) the child has earned the reward.

The last activity is a reward that consists of a physical fluorescent star, which the child can place on a laminated A4-sized paper chart as seen on Figure 2 and further described in [23].

The MOBERO morning module is similar to the bedtime module in that it displays a structured list of activities. However, this module does not contain any activities for the parents and the child is furthermore rewarded with virtual medals for completing the activities within a certain timeframe (e.g., four medals for brushing teeth in two minutes) as seen on Figure 1.

From the first one-month phase of our study (baseline and intervention periods), we found that MOBERO significantly lowered the parents' frustration level during morning and bedtime routines and the parents reported their child to be more independent during both morning and bedtime routines [23]. These findings were based on an analysis of parents' daily assessments throughout both the baseline and intervention periods. Furthermore, we found an improvement in the children's sleep habits based on a validated child sleep screening questionnaire [16], and a reduction in parent reported ADHD symptoms based on the ADHD Rating Scale (ADHD-RS) [7], a validated rating scale used to measure the severity of ADHD symptoms. In contrast to our expectations, we did not find any statistically significant change in the parent reported bedtime, sleep time or sleep delay (the time it takes the child to fall asleep).

\section{STUDY DESIGN}

The first phase of our MOBERO study was designed to investigate the potential of using MOBERO to assist in establishing healthy morning and bedtime routines [23]. This phase was divided into a two-week baseline period and a two-week intervention period, in which the families used MOBERO during morning and bedtime activities.

In the second phase of the study (the month following the end of the intervention period), we wanted to investigate what happened after MOBERO was no longer used in the families, as this provides insights into long-term effects of MOBERO on the family practices. Therefore, the provided smartphone on which MOBERO was installed was taken away from the families at the end of the two-week intervention period.

\section{Participants}

13 children with ADHD and their families participated in the study. All children were clinically diagnosed with ADHD and had an ADHD-RS score within the expected gender and age stratified normative data scores for children clinically diagnosed with ADHD [31]. Seven children received medical treatment, however no changes were made to the children's medication during either the baseline, intervention or follow-up phases of our study.

\section{Conducted Interviews}

For this paper we include interviews conducted at the end of the intervention period as well as interviews from the one-month follow up phase. Unfortunately, due to scheduling issues around the start of school summer holidays, only four families participated in the one-month follow-up interview (mean duration $=14$ minutes). However, all families did complete the MOBERO-Q questionnaire introduced in the next sub-section. We have decided to include the interviews with these four families, as they all had quite different experiences after the onemonth period following the intervention period, which highlight how heterogeneous families of children with ADHD can be and how differently technology affects these families. We did not select the four families, instead they were simply the four families who were available for an interview after the one-month follow-up period.

\section{Questionnaire Measures}

All 13 families completed a custom made questionnaire (MOBERO-Q) both prior to the baseline period, after the intervention period, and one-month after the end of the intervention period. The questions in MOBERO-Q were related the child's sleep habits, and activities around their bedtime, and all answers were recorded on a five-point Likert scale ranging from 5: "Very often (five to seven times a week)" to 1: "Very rarely (less than one time per half year)" as well as allowing parents the opportunity to submit general text comments. In the Findings section below all MOBERO-Q questions are presented and analyzed.

\section{Data Analysis}

\section{MOBERO-Q Questionnaire Processing}

Because the parent responses in MOBERO-Q were ordinal and represented three repeated measures (prior to the baseline period, after the intervention period, and one month after the intervention period), we used a Friedman test to investigate if a statistical significance existed between the three groups; followed by a two-tailed Wilcoxon Signed Rank post-hoc test. 

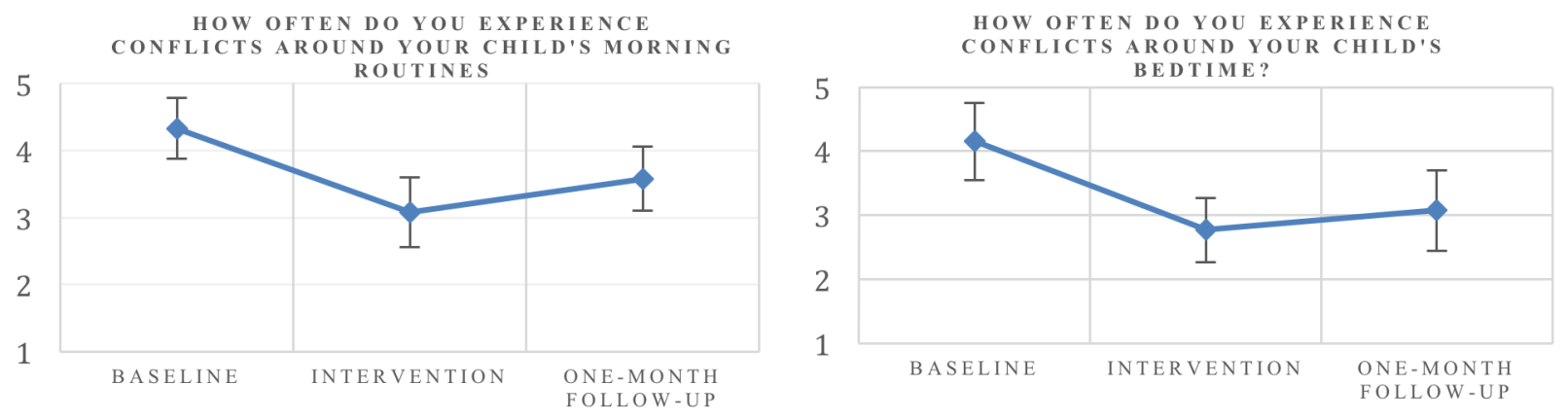

Figure 3: Graphs showing the MOBERO-Q conflict level during morning (left) and bedtime (right) routines prior to the baseline period, after the intervention period, and one month after the intervention period. The y-axis represents a five-point

Likert scale where $5=$ "Very often (five to seven times a week)" and $1=$ "Very rarely (less than one time per half year)"

\section{Interview Processing}

All audio-recorded interviews were conducted, transcribed and thematically analyzed in Danish [4], and selected passages were translated to English for inclusion in this paper. All names and personal information have been removed to preserve anonymity.

\section{FINDINGS}

Parents Still Report Fewer Conflicts Around Morning and Bedtime Routines

Parent reported conflict level around morning routines showed a statistically significant difference between the three MOBERO-Q tests (baseline, intervention, and onemonth follow-up), $\chi^{2}(2)=13.6, p<0.01$, with significant differences between baseline $(\mathrm{Mdn}=4)$ and intervention $(\mathrm{Mdn}=3)$ responses, $\mathrm{z}=-2.8, \mathrm{p}<0.01$; and between baseline and one-month follow-up $(\mathrm{Mdn}=3)$ responses, $\mathrm{z}=$ 2.4, $\mathrm{p}<0.05$; but not between intervention and one-month follow-up responses, $\mathrm{z}=-1.9, \mathrm{p}>0.05$. Similarly, the parent-reported conflict level around bedtime routines showed a significant difference between the three groups, $\chi^{2}(2)=8.1, p<0.5$, with significant differences between baseline $(\mathrm{Mdn}=5)$ and intervention $(\mathrm{Mdn}=2)$ responses, $\mathrm{z}$ $=-2.6, \mathrm{p}<0.01 ;$ and baseline and one-month follow-up $(\mathrm{Mdn}=3)$ responses, $\mathrm{z}=-2.4, \mathrm{p}<0.05$; but not between intervention and one-month follow-up responses, $z=-0.7, p$ $>0.05$. This suggest that the reduction effect of parents' experienced conflict level during both morning and bedtime routines was sustained one month after ending the MOBERO intervention period as illustrated in Figure 3.

Parents Still Report Lower Frustration Levels During Morning and Bedtime Routines

Parent-reported frustration level around morning routines showed a statistically significant difference between the three groups, $\chi^{2}(2)=12.4, p<0.01$, with statistically significant differences between baseline $(\mathrm{Mdn}=4)$ and intervention $(\mathrm{Mdn}=3$ ) responses, $\mathrm{z}=-2.8, \mathrm{p}<0.01$; and baseline and one-month follow-up ( $\mathrm{Mdn}=3$ ) responses, $\mathrm{z}=$ $-2.2, \mathrm{p}<0.05$; but no significant difference between intervention and one-month follow-up responses, $z=-1.9, p$ $>0.05$. Similarly, the parents' reported frustration level around bedtime routines showed a significant difference between the three groups, $\chi^{2}(2)=6.2, p<0.05$, with significant differences between baseline $(\mathrm{Mdn}=4)$ and intervention $(\mathrm{Mdn}=3$ ) responses, $\mathrm{z}=-2.4, \mathrm{p}<0.05$; and with significant differences between baseline and the onemonth follow-up responses $(\mathrm{Mdn}=3), \mathrm{z}=-2.1, \mathrm{p}<0.05$; however there was no difference between intervention and one-month follow-up responses, $\mathrm{z}=-0.8, \mathrm{p}>0,05$. This suggests that the lowering effect of MOBERO on the parents' frustration level during morning and bedtime routines continued one-month after end of the intervention phase, compared to the baseline phase, indicating a longterm effect of using MOBERO.

MOBERO Did Allow for a Fresh Start, However Some Families Found It Difficult to Sustain the Effect

As suggested by the statistical analysis of parents' MOBERO-Q responses of their experienced frustration and conflict levels during morning and bedtime routines, most families continued to benefit from the techniques and strategies offered by the MOBERO prototype. This is supported by a comment from one family: "[...] the afternoons have actually, for some reason, continued to be reasonable. It is as if the phone [MOBERO] has started something, so that we now actually do not experience as many conflicts around the bedtime routines". For some, MOBERO did allow for a new start as exemplified by: "[ ... ] for him, it was also a break, which meant that he could start over in a new way". Interestingly, one family reported that they experienced that they returned to baseline conditions almost right away, another reported that "I think she gradually forgot it over 14 days and then it was over, and now we can hardly get her in bed", and yet another family did experience a fundamental change in their child's morning and bedtime routines: "I also think that the routines have maybe been more clear for him [in MOBERO], and that he should do one thing at a time, and that is fine. Some of the issues before were that he would get stressed that he should both change clothes, brush teeth, and so on [...]. So [now] he follows the activities as they were in the phone [MOBERO] - we did have the same activities before, however I think that it [MOBERO] has made them a little clearer for him [...]." Though, the 
statistical analysis of MOBERO-Q showed that most families did report a positive effect of MOBERO on frustration and conflicts levels one month after the end of the intervention period, it is interesting how different the four interviewed families experienced the time after they handed MOBERO back.

Unexpected Consequences of MOBERO on Family Practices as Parents Enforced Too Strict Routines

Our expectations about the effect of MOBERO in the first phase of our study were that it would lower the parents' frustration level and improve the child's independence level. As we have reported [23] this was found to be the case overall; however, in two families, we experienced almost the opposite effect.

The first family summed up their experience of the intervention period like this: "He has been mad at it [MOBERO], he has been mad at you and he has been mad at us. 'Why are we destroying his life?' I think we have been called child abusers and animal abusers and a lot of other things [...]. It turned out, that the bedtime routine this family had created in MOBERO was very far from their existing routine, and that the family enforced this new routine with a strict and zero discussion policy e.g., "[before] he had it as the yolk of an egg [...] he sat in there [in his bed] with his iPad and ate - he is not allowed to do that anymore. [...] So there are many privileges that have been taken totally away from him (the mother interrupts): Yes, maybe that was too many changes at once.". Previously, the parents had not been able to provide sufficient structure for their child, so it seemed that when they were given the opportunity to get external help (from MOBERO) to provide this, they chose a very strict structure and consequence strategy, which simply was too drastic a change for the child. In the other family, we found almost the same issue: the parents had demanded too much of a change in the child's daily routines, and again we observed a resistance from the child to using MOBERO, which resulted in increased parent reported conflicts in the intervention period.

\section{Challenges in Keeping Consistent Bedtime Routines One Month After the use of MOBERO.}

To the MOBERO-Q question "How often do you follow a consistent bedtime routine?" we observed a statistically significant difference between the three groups, $\chi^{2}(2)=8.1$, $\mathrm{p}<0.5$. Interestingly, we do not see a significant difference in parent's responses between baseline $(\mathrm{Mdn}=5)$ and intervention $(\mathrm{Mdn}=5), \mathrm{z}=-0.7, \mathrm{p}>0.05$; In contrast, we do see a statistical significant change between both baseline and one-month follow-up $(\mathrm{Mdn}=4)$ responses, $\mathrm{z}=-2.0, \mathrm{p}$ $<0.05$, and between intervention and one-month follow-up responses, $\mathrm{z}=-2.3, \mathrm{p}<0.05$. This suggest that in the month following the end of intervention period, the families did not have as consistent bedtime routines as both before and during the use of MOBERO.
The Children are Still Getting Too Little Sleep and Have Challenges Waking up in the Morning

We did not record a statistically significant difference in the parents' response to the MOBERO-Q question "How often do you experience that your child is not getting enough sleep?" between the three groups, $\chi^{2}(2)=4.1, p=0.1$. These findings are in line with our findings from the main study, where our analysis of the parent interviews revealed that the children still had trouble falling asleep after using MOBERO [23]. Similarly, we did not see a statistically significant difference in the parents' response to the MOBERO-Q question "How often do you experience that your child has difficulties getting up in the morning?" $\chi^{2}(2)$ $=5.2, \mathrm{p}=0.08$. However, in both cases we did observe a positive change in median score: baseline $(\mathrm{Mdn}=4)$, intervention $(\mathrm{Mdn}=3)$ and one-month follow-up ( $\mathrm{Mdn}=$ $3)$.

\section{LIMITATIONS AND FUTURE WORK}

We are aware that a limitation of our study is the low number of participating families, and especially that we only managed to conduct interviews with four of these families due to scheduling issues around the start of the school summer holidays. Therefore, we have also been careful not to over-generalize our quantitative findings. Similarly, we have chosen to only report the clearest statements from our one-month follow-up interviews. Another potential limitation is that for several of the families, the one-month follow-up phase included one or more weeks of school holiday, which could have had an affect on the family practices, including morning and bedtime routines. We are currently planning to conduct a longitudinal study of an enhanced version of MOBERO in families of children with ADHD. As future work, we plan to explore the potential of technologies to support families to establish and improve sleep habits for their children as we have discussed how important healthy sleep habits are.

\section{DISCUSSION}

Building on the findings from the first phase of our study reported in [23], this second phase follow-up study suggest that MOBERO supported the families in establishing sound family practices that were still measurable one month after the families had handed MOBERO back to us. Similarly to our findings, Anastopoulos et al. investigated the effect of parent training on parent functioning in families of children with ADHD, and found that over a two-month follow-up period parents reported general improvements in parent functioning related to reduced stress and enhanced selfesteem [1]. However, it is interesting that the four families we managed to interview reported very different experiences in the one month following the intervention period. We have not been able to give a "clear" explanation as to why this is the case based on the collected data from our studies. However, from our experiences from interacting with and getting to know these families, we can only speculate on why they behaved as they did. These speculations are related to the general level of structure in 
the families prior to our study, their expectations to the technology (MOBERO), the severity of the child's ADHD diagnosis (and potential co-morbid disorders), and the parents' understanding and ability to cope with their child's challenges. However, these are only speculations, and we hope that we in future work can learn more about the family practices that cause these differences, such that we design our technologies in the best way to support education and prolonged effects for parents and their children with ADHD.

\section{Clarifying the Reported Drop in Bedtime Consistency}

As presented in the findings section, our analysis suggests that the families found it harder to stick to consistent bedtimes routines in the month after having used MOBERO, compared to both before and during the first phase of our study. We believe this finding may be flawed for two reasons. First, for a few of the families, the fact that the second phase of the study included one or more weeks of school summer holidays may have caused the parents to have a less strict bedtime routine, which is supported by the increase in median score. Second, our interviews with the families prior to baseline period and their daily assessment from the baseline period suggest that the families actually did not have very consistent bedtime routines [23], in contrast to MOBERO-Q responses prior to the baseline period.

\section{The Value of Follow-up Studies}

Several researchers within the IDC community have conducted longitudinal studies and reported their benefits and challenges (e.g., $[9,13,14,26,27]$ ). We agree that longitudinal studies are of high importance as these provide insights into how participants interact with and adapt technology over time. As we have described in the future work section, this is also part of the reason why we are planning to conduct a longitudinal study of MOBERO. However, in contrast to longitudinal studies, follow-up studies can provide insights into how users change, adapt and feel after they can no longer use the technology. As discussed above, we would never have learned about how differently the family practices changed after MOBERO was no longer being used. Had we had the opportunity to interview all families, we might have been able to identify why the families developed so differently. Such knowledge would be of importance before we design and conduct our longitudinal study, as there might be issues under the surface that we did not uncover from the first phase of our study.

Another value of conducting follow-up studies in our case, is that we can identify that the positive impact MOBERO had on the families continued even one month after they handed it back to us. This shows us, that not only did MOBERO help during the two-week intervention period, but it also gave the families techniques that they continued to benefit from even after the technology was no longer there. It would be interesting to conduct additional follow- up studies to investigate if and how the family practices develop over longer time. However, as we have not been able to find much work on this topic, we also invite researchers to begin to discuss thoughts and best practices for setting up and conducting follow-up studies.

Finally, follow-up studies are a way to also consider the ethics of taking away technologies that appear to be a real help for the user and which the user has come to depend on in everyday life. A lot of work within IDC and HCI in general focuses on evaluating assistive technologies and reporting results from these studies (e.g., [11,25]). However, what happens when this technology is taken away? Do we as researchers who work with such a vulnerable population as children, have an extra responsibility to investigate how our technology affects the children after it is no longer deployed? In our case, we wanted to make sure that MOBERO did not do that, so that we can continue to conduct future studies.

\section{CONCLUSION}

In this paper we have reported the findings of a one-month follow-up study of MOBERO, a mobile system that supports families of children with ADHD to change their family practices around morning and bedtime routines. Our findings suggest that the positive changes in family practices related to parent rated frustration and conflict level around their child's morning and bedtime routines, which MOBERO established in the intervention period, still positively affected the families one month after MOBERO had been taken away. However, from our one-month follow-up interviews, we also learned how MOBERO affected the practices of the families quite differently in the follow-up period. Finally, we discussed the value of conducting follow-up studies and provided insights into family practices relevant for future research on technologies for the ADHD domain.

\section{SELECTION AND PARTICIPATION OF CHILDREN}

16 children with ADHD were recruited for this study, however only 13 children were selected to be included in the study based on several predefined inclusion criteria, which required answering a screening questionnaire, that the child should be clinically diagnosed with ADHD or under investigation for ADHD, that the child had an ADHD-RS score within the ADHD range, that the child should not be below the age of six, and that the child should not have a condition other than ADHD as the primary diagnosis. After the inclusion selection, the parents were introduced to the study design in their home by the first author. Prior to the study ethical approval was obtained from the regional ethical committee.

\section{Acknowledgements}

We thank all of the families for participating in our study. Furthermore, we want to thank Center for ADHD, Lene Ruge Møller, Lotte Fensbo, Line Møller Sletting, Anne Virring Sørensen, and Per Hove Thomsen for their valuable insights and feedback. 


\section{REFERENCES}

1. Arthur D. Anastopoulos, Terri L. Shelton, George J. DuPaul, and David C. Guevremont. 1993. Parent training for attention-deficit hyperactivity disorder: Its impact on parent functioning. Journal of Abnormal Child Psychology 21, 5: 581-596. http://doi.org/10.1007/BF00916320

2. Jared S. Bauer, Sunny Consolvo, Benjamin Greenstein, et al. 2012. ShutEye: Encouraging Awareness of Healthy Sleep Recommendations with a Mobile, Peripheral Display. Proceedings of the SIGCHI Conference on Human Factors in Computing Systems, ACM, 1401-1410. http://doi.org/10.1145/2207676.2208600

3. Joseph Biederman and Stephen V Faraone. 2005. Attention-deficit hyperactivity disorder. The Lancet 366, 9481: 237-248. http://doi.org/10.1016/S01406736(05)66915-2

4. Virginia Braun and Victoria Clarke. 2006. Using thematic analysis in psychology. Qualitative Research in Psychology 3, 2: 77-101. http://doi.org/10.1191/1478088706qp063oa

5. Søren Dalsgaard, Preben Bo Mortensen, Morten Frydenberg, and Per Hove Thomsen. 2013. Long-term criminal outcome of children with attention deficit hyperactivity disorder. Criminal behaviour and mental health: $\quad$ CBMH 23, 2: 86-98. http://doi.org/10.1002/cbm.1860

6. Julia F. Dewald, Anne M. Meijer, Frans J. Oort, Gerard A. Kerkhof, and Susan M. Bögels. 2010. The influence of sleep quality, sleep duration and sleepiness on school performance in children and adolescents: A metaanalytic review. Sleep Medicine Reviews 14, 3: 179189. http://doi.org/10.1016/j.smrv.2009.10.004

7. Douglas E. Faries, Ilker Yalcin, Donald Harder, and John H. Heiligenstein. 2001. Validation of the ADHD Rating Scale as a clirlician administered and scored instrument. Journal of Attention Disorders 5, 2: $107-$ 115. http://doi.org/10.1177/108705470100500204

8. Michael W. Firmin and Annie Phillips. 2009. A Qualitative Study of Families and Children Possessing Diagnoses of ADHD. Journal of Family Issues 30, 9: 1155-1174. http://doi.org/10.1177/0192513X09333709

9. Elizabeth Foss, Allison Druin, and Mona Leigh Guha. 2013. Recruiting and Retaining Young Participants: Strategies from Five Years of Field Research. Proceedings of the 12th International Conference on Interaction Design and Children, ACM, 313-316. http://doi.org/10.1145/2485760.2485798

10.Reut Gruber, Sabrina Wiebe, Lisa Montecalvo, Bianca Brunetti, Rhonda Amsel, and Julie Carrier. 2011. Impact of Sleep Restriction on Neurobehavioral Functioning of Children with Attention Deficit Hyperactivity Disorder. Sleep 34, 3: 315-323.

11.Gillian R. Hayes and Stephen W. Hosaflook. 2013. HygieneHelper: Promoting Awareness and Teaching Life Skills to Youth with Autism Spectrum Disorder.
Proceedings of the 12th International Conference on Interaction Design and Children, ACM, 539-542. http://doi.org/10.1145/2485760.2485860

12. Matthew Kay, Eun Kyoung Choe, Jesse Shepherd, et al. 2012. Lullaby: A Capture \& Access System for Understanding the Sleep Environment. Proceedings of the 2012 ACM Conference on Ubiquitous Computing, ACM, 226-234. http://doi.org/10.1145/2370216.2370253

13.Olga Lyra, Evangelos Karapanos, Rúben Gouveia, Valentina Nisi, and Nuno J. Nunes. 2013. Engaging Children in Longitudinal Behavioral Studies Through Playful Technologies. Proceedings of the 12th International Conference on Interaction Design and Children, ACM, 396-399. http://doi.org/10.1145/2485760.2485817

14. Regan L. Mandryk, Shane Dielschneider, Michael R. Kalyn, et al. 2013. Games As Neurofeedback Training for Children with FASD. Proceedings of the 12th International Conference on Interaction Design and Children, ACM, 165-172. http://doi.org/10.1145/2485760.2485762

15.Greta M Massetti, Benjamin B Lahey, William E Pelham, et al. 2008. Academic achievement over 8 years among children who met modified criteria for attentiondeficit/hyperactivity disorder at 4-6 years of age. Journal of abnormal child psychology 36, 3: 399-410. http://doi.org/10.1007/s10802-007-9186-4

16.J. A. Owens, A. Spirito, and M. McGuinn. 2000. The Children's Sleep Habits Questionnaire (CSHQ): psychometric properties of a survey instrument for school-aged children. Sleep 23, 8: 1043-1051.

17.Ruth Perou, Rebecca H. Bitsko, Stephen J. Blumberg, et al. 2013. Mental health surveillance among childrenUnited States, 2005-2011. MMWR Surveill Summ 62, Suppl 2: 1-35.

18.Guilherme Polanczyk, Maurício Silva de Lima, Bernardo Lessa Horta, Joseph Biederman, and Luis Augusto Rohde. 2007. The worldwide prevalence of ADHD: a systematic review and metaregression analysis. The American Journal of Psychiatry 164, 6: 942-948. http://doi.org/10.1176/ajp.2007.164.6.942

19.Ruth Segal. 1998. The Construction of Family Occupations: A Study of Families with Children Who Have Attention Deficit/Hyperactivity Disorder. Canadian Journal of Occupational Therapy 65, 5: 286292. http://doi.org/10.1177/000841749806500506

20.Dorothé Smit and Saskia Bakker. 2015. BlurtLine: A Design Exploration to Support Children with ADHD in Classrooms. In Human-Computer Interaction INTERACT 2015, Julio Abascal, Simone Barbosa, Mirko Fetter, Tom Gross, Philippe Palanque and Marco Winckler (eds.). Springer International Publishing, 456460. Retrieved October 6, 2015 from http://link.springer.com/chapter/10.1007/978-3-31922723-8_37 
21.Tobias Sonne and Kaj Grønbæk. 2015. Designing Assistive Technologies for the ADHD Domain. In Pervasive Computing Paradigms for Mental Health. Springer International Publishing, 259-268. Retrieved September 14, 2015 from http://doi.org/10.1007/978-3319-32270-4 26

22.Tobias Sonne and Mads Møller Jensen. 2016. ChillFish: A Respiration Game for Children with ADHD. Proceedings of the TEI '16: Tenth International Conference on Tangible, Embedded, and Embodied Interaction, ACM, 271-278. http://doi.org/10.1145/2839462.2839480

23.Tobias Sonne, Jörg Müller, Paul Marshall, Carsten Obel, and Kaj Grønbæk. 2016. Changing Family Practices with Assistive Technology: MOBERO Improves Morning and Bedtime Routines for Children with ADHD. Proceedings of the 34th Annual ACM Conference on Human Factors in Computing Systems, ACM. http://doi.org/10.1145/2858036.2858157

24.Tobias Sonne, Carsten Obel, and Kaj Grønbæk. 2015. Designing Real Time Assistive Technologies: A Study of Children with ADHD. Proceedings of the Annual Meeting of the Australian Special Interest Group for Computer Human Interaction, ACM, 34-38. http://doi.org/10.1145/2838739.2838815

25.Abigale Stangl, Jeeeun Kim, and Tom Yeh. 2014. 3D Printed Tactile Picture Books for Children with Visual Impairments: A Design Probe. Proceedings of the 2014 Conference on Interaction Design and Children, ACM, 321-324. http://doi.org/10.1145/2593968.2610482

26. Arnold P. O. S. Vermeeren, Effie Lai-Chong Law, Virpi Roto, Marianna Obrist, Jettie Hoonhout, and Kaisa Väänänen-Vainio-Mattila. 2010. User Experience Evaluation Methods: Current State and Development Needs. Proceedings of the 6th Nordic Conference on Human-Computer Interaction: Extending Boundaries, ACM, $521-530$ http://doi.org/10.1145/1868914.1868973

27.Jorick Vissers, Lode De Bot, and Bieke Zaman. 2013. MemoLine: Evaluating Long-term UX with Children. Proceedings of the 12th International Conference on Interaction Design and Children, ACM, 285-288. http://doi.org/10.1145/2485760.2485836

28.Peter M. Wehmeier, Alexander Schacht, and Russell A. Barkley. 2010. Social and Emotional Impairment in Children and Adolescents with ADHD and the Impact on Quality of Life. Journal of Adolescent Health 46, 3: 209-217. http://doi.org/10.1016/j.jadohealth.2009.09.009

29. Orad Weisberg, Ayelet GalOz, Ruth Berkowitz, et al. 2014. TangiPlan: Designing an Assistive Technology to Enhance Executive Functioning Among Children with Adhd. Proceedings of the 2014 Conference on Interaction Design and Children, ACM, 293-296. http://doi.org/10.1145/2593968.2610475

30.Attention deficit hyperactivity disorder | introduction Guidance and guidelines | NICE. Retrieved December
10 , 2015 from http://www.nice.org.uk/guidance/cg72/chapter/introducti on

31. Validity and clinical feasibility of the ADHD rating scale (ADHD-RS) A Danish Nationwide Multicenter Study - Szomlaiski - 2008 - Acta Pædiatrica - Wiley Online Library. Retrieved September 9, 2015 from http://onlinelibrary.wiley.com/enhanced/doi/10.1111/j.1 651-2227.2008.01025.x/ 